\title{
Management System of Teaching Resource Based on SSH Framework
}

\author{
Liangtao Yang \\ Shanghai Second Polytechnic University \\ Shanghai, China \\ E-mail:ltyang@pc.sspu.cn
}

\begin{abstract}
According to the brief introduction of the three frameworks----Spring, Struts and Hibernate, the paper constructs a teaching resource management system by using SSH framework based on J2EE. Such a system architecture reduces the coupling system code and improves system maintainability and extensibility.
\end{abstract}

Key words-SSH Framework; Teaching Resource; Management System

\section{INTRODUCTION}

With the continuous advance of the education informationalization, teaching resources in the network is growing rapidly. It is very important to manage decentralized, disorderly teaching resources effectively to allow users to use them to study and work conveniently and efficiently. It is an imperative problem to establish a teaching resource management system [1-2], which will make teaching resources systematic and scientific, and constitute a unified teaching resources library to provide content-rich teaching resources for the user, and achieve the teaching resources standardized storage and management as well as excellent teaching resource sharing. From the practical point of view, this paper designs a management system of teaching resources based on J2EE technology and Struts + Spring + Hibernate framework architecture, and at the same time the paper discusses some technology about the management system.

\section{SSH FRAMEWORK}

SSH Framework, as a reusable software, can shorten the application development cycle, improve development quality and increase the maintainability of the system. The $\mathrm{SSH}$ architecture is the widest integration development model in J2EE enterprise application. SSH architecture integrates Struts, Spring and Hibernate three major open-source framework [3-5]. They are respectively used in the presentation layer, business logic and data persistence layer to achieve a decoupling between layers, with highly scalable, high performance and high security features.

\section{A. presentation layer and Struts framework}

Struts is the development of open-source framework based on Model (Model) - the view (View) - Controller (Controller) (MVC) pattern application framework. It is a good combination to Jsp, Java Servlet, Java Bean, Taglib and other technology. In the Struts framework, the model consists of the ActionForm Bean and other business logic Java Bean or EJB component composition. The controller is moved by ActionServlet. The view is made of a set of JSP files. Struts framework uses the controller to separate the model and the view, user interface and business logic separation, which greatly reduces development time, in large part to get rid of the shackles of the traditional development pattern, improve the system's flexibility, reusability and maintainability. Their specific implementation is shown in Figure 1.

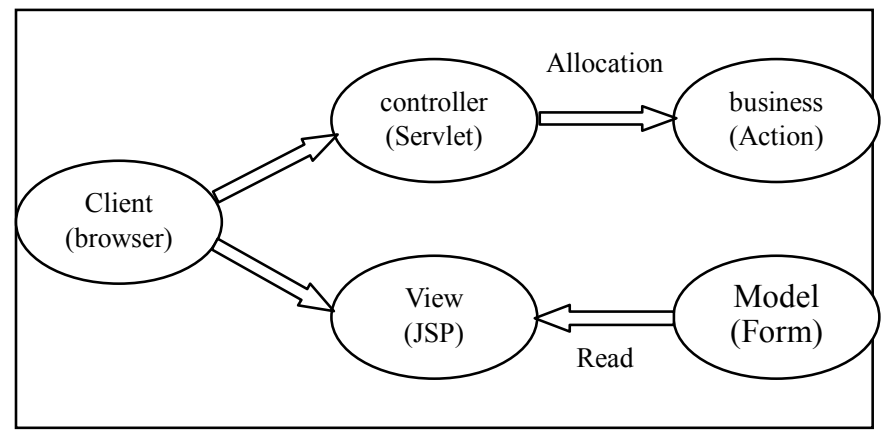

Figure 1. Struts Framework Structure

\section{B. business logic layer and Spring framework}

Business Logic Layer (Business Logic Layer) is responsible for encapsulating complex business logic, performs the business logic according to the presentation layer pass and returns business results to the presentation layer. Spring is a lightweight J2EE application framework. It reduces the degree of coupling between business components and enhances system scalability by Inversion of Control (IOC) and facing to the aspects of programming (AOP) applications and oriented programming. The Spring Framework is a layered architecture, one of the most important module is the core container. The core container defines creating, configuring and the way of managing Bean It provides the basic functionality of the Spring Framework. The main components of the core container is the BeanFactory, it is the realization of the factory pattern. BeanFactory uses Inversion of Control (IOC) mode to separate the application configuration and dependency specification from the actual application code.

\section{C. data persistence layer and Hibernate framework}

The data persistence layer is responsible for completion of the various data persistence programming and also serves for the system logic layer. It provides data access methods to help other programmers avoid re-write applications to access data persistence layer (Persistent layer) in order to focus on 
business logic development and can reuse mapping framework in different projects, greatly simplifying the development process of data additions and deletions repair check function, without the loss of the natural advantages of the multi-layer structure. Hibernate is an ORM mapping tool and the JDBC lightweight object package. It can be used in any occasion of JDBC. Hibernate not only provides mapping from Java classes to the data table but also provides data query and recovery mechanisms. For using JDBC and SQL, Hibernate can greatly reduce the workload of operating the database.

\section{FUNCTIONAL MODULE DESIGN OF THE SYSTEM}

Teaching resources management system is based on management innovation, teaching resources management as the core, material resource library, teaching courseware library and network courses library for support It is also the platform to serve the teaching purpose-built shared learning resource center. The system goal is to realize the scientific classification and centralized management of learning resources, improve resource sharing and learning platform interoperability to make teaching resources serve the teaching process better.

The system users include ordinary users, auditors and administrators. Ordinary users can upload resources, retrieve resources, browse resources, download resources and evaluate resources. The auditor can retrieve the resources and can check and publish the uploaded resources by users. The administrators have the highest authority to retrieve and manage resources, manage users and the entire system, etc. The systems architecture design is shown in Figure 2.

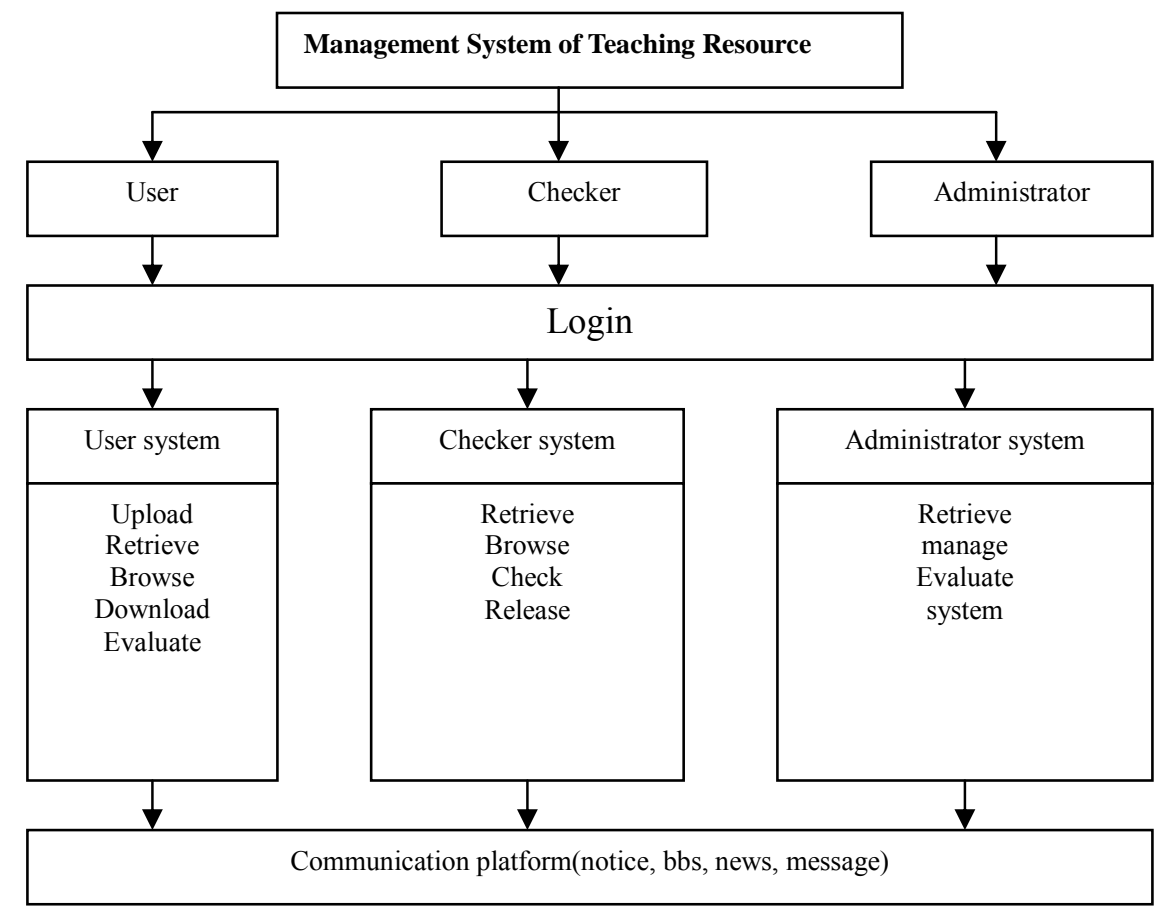

Figure 2. Overall system diagram

\section{SYSTEM ARCHITECTURE DESIGN AND IMPLEMENTATION}

\section{A. system architecture design}

The system uses J2EE architecture based the MVC pattern [6-8] to realize the B/S multi-tier Web application. It is divided into the presentation layer, business logic layer, data persistence layer and database layer. Each layer in the application has a clear responsibility. The rational allocation $\&$ combination of Struts, Spring and Hibernate can make layers flexible and clear to enhance the reusability and maintainability of the system. Figure 3 is the system hierarchical chart based on Struts, Hibernate and Spring Framework. In Figure 3, Struts Layer is responsible for the Web tier and process control. ActionFormBean receives submission data from the web page and processes it through
Action and then Forward to the corresponding page. Action is defined in struts-config.xml and loaded by ActionServlet. Spring Layer is responsible for the management of the business layer, business logic processing and circulation. Business Service provides Action with a unified interface calls to encapsulate data access object DAO of the persistence layer. Hiberante Layer is responsible for data persistence and encapsulates the underlying operating on the back-end database.

In $\mathrm{SSH}$ framework system, the basic call flow of the object is JSP $\rightarrow$ Action $\rightarrow$ Business Service $\rightarrow$ DAO $\rightarrow$ Hibernate. Data flow is that ActionFormBean accepts users data and Action will take the data from the ActionFormBean to seal in the package as VO or PO and then call the business layer Bean class to Forward after a variety of business processes. The service layer beans will call the 
DAO interface method for persistence operations after receipt of the PO object.

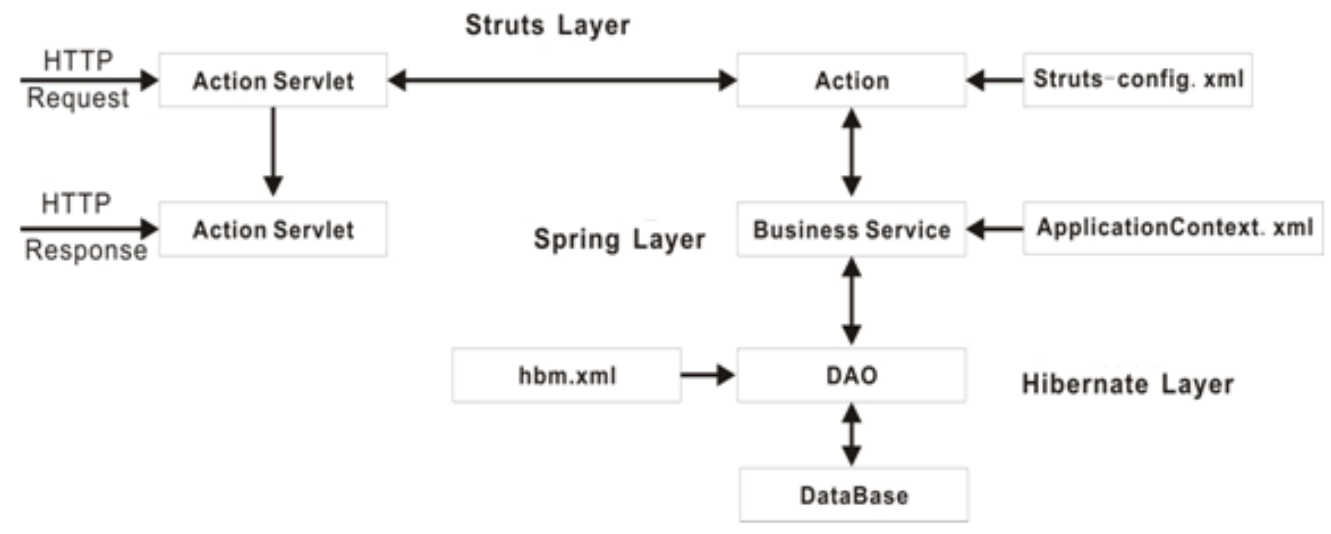

Figure 3. System architecture diagram based on SSH framework

\section{B. implementation of the system}

The presentation layer of the system is realized by Struts. The configuration files based on XML, Struts-config.xml link the model, view and controller. Controller function is implemented by ActionServlet. ActionServlet's core is concentrated the configuration file struts-config.xml defined by the navigation on all pages, which includes the statement of each business client request, the Action class of dealing with the request, ActionForm class of collecting the data information from the request and instructs the view which should return to the users after the operational implementation. Business Logic Layer uses Spring Framework. It is mainly responsible for processing the application's business logic. The business logic layer receives the users' requests of the presentation layer, controls the flow of application logic and completes data access operations through the persistence layer. In the persistence layer, Hibernate offers HQL operating entity object to realize the function of the database ' add, delete, change, check', and transfers the operation of the database tables directly into the operation of the object, so as to achieve the purpose of operating the database.

The following uses the user management in the administrator subsystem as an example to particularly analyze the Struts + Hibernate + Spring multi-frame applied in the system. The main function of this part is to add or delete the users and modify their permissions. For example, to add a system user, enter user information and submit, by Struts call AddUserAction's execute method, the business layer service interface implementation class, and then indirectly invoke the DAO interface implementation class. The DAO interface implementation class encapsulates the corresponding persistent objects via Hibernate to access the data table CRUD operations. The following gives the part code segment of the Spring applicationContext.xml configuration file:

\footnotetext{
$<$ beans $>$

$<$ bean name $=$ "/AddUserAction" class =

"user.action.AddUserAction"

singleton $=$ "false" >
}

$$
\begin{aligned}
& <\text { property name }=\text { "userServices" }> \\
& <\text { ref local }=\text { "UserServices" }></ \text { ref }> \\
& <\text { /property }> \\
& <\text { /bean }>
\end{aligned}
$$

Through configuration code above, the spring will connect strutsAction which is responsible for the interfacial layer with userSerivces which is responsible for the service layer. StrutsAction sets userServices in its own code by Spring dependency injection.

Struts use struts-config.xml as the configuration file. In this configuration file, struts configurate the Spring components, DelegatingRequestProcessor to let Spring manage individual components. Code as follows:

$<$ controller processorClass $=$

"org.springframework.web.struts.DelegatingRequestProce ssor"/>

\section{Conclusion}

SSH framework is the most popular integration architecture design in current Java technology platform. Through separation of decoupling between the presentation layer, business logic layer and data persistence layer, the development mode simplifies the system development. It is also easy to manage and maintain and improves the system development efficiency with broad application prospects. The paper uses struts + spring + hibernate technology to conduct system architecture, develops teaching resources management system based on J2EE platform, realizes the unified management of teaching resources, resource sharing, resource exchange and improves the utilization of teaching resources with a certain application value.

\section{REFERENCES}

[1] Jiangang Cheng, Liangchun He. Design and Realization of Distributed Education Resource Database Based on Web [J].E-education Research,2004(11):25-27.

[2] Ru Xu.[J].Design and Realization of Education Resource Management System Based on ASP.NET[J]China Education Info,2006(7):46-48. 
[3] Wei Jie, Shuang Zhang. Proficient in struts - java server, framework, tools and integrated fashion[M].BeiJing: The People's Posts and Telecommunications Press,2006.

[4] Shifei Luo. Proficient in Spring[M].BeiJing: Electronic Industry Press, 2005.

[5] Guohui Wang,Wenqiang Ma.Complete Guide to Hibernate application development[M]. The People's Posts and Telecommunications Press, 2006.
[6] Yue Wu,Jingnong Weng. Building strategy for web applications combined struts with hibernate[J].Computer Engineering and Design,2006,27(7):1176-1179.

[7] Weidong Li,Huaji Shi,Xingyi Li.Study of enterprise application integration based on web services on J2EE Platform[J].Computer Engineering and Design,2005(261):153-155.

[8] Gang Li. Lightweight J2EE Enterprise Application combat: Struts + Spring + Hibernate Integration Development[M].BeiJing:Electronic Industry Press, 2007. 